\title{
Evolution of stellar-gaseous disks in cosmological halos
}

\author{
A. Curir ${ }^{1}$, P. Mazzei ${ }^{2}$, and G. Murante ${ }^{1}$ \\ 1 INAF-Osservatorio Astronomico di Torino. Strada Osservatorio 20, 10025 Pino Torinese (Torino), Italy \\ e-mail: curir@oato.inaf.it \\ 2 INAF-Osservatorio Astronomico di Padova. Vicolo Osservatorio 5, 35122 Padova, Italy \\ e-mail: paola.mazzei@oapd.inaf.it
}

Received 27 March 2006 / Accepted 12 January 2007

\begin{abstract}
Aims. We explore the growth and evolution of the bar instability in stellar-gaseous disks embedded in a suitable dark matter halo evolving in a fully consistent cosmological framework. The aim of this paper is to point out the impact of different gas fractions on the bar formation, inside disks of different disk-to-halo mass ratio, and the role of the cosmological framework.

Methods. We performed cosmological simulations with the same disk-to-halo mass ratios as in a previous work where the gas was not taken into account. We compared results of the new simulations with the previous ones to investigate the effect of the gas by analysing the morphology of the stellar and gaseous components, the stellar bar strength, and the behaviour of its pattern speed.

Results. In our cosmological simulations, inside dark matter dominated disks, a stellar bar, lasting $10 \mathrm{Gyr}$, is still living at $z=0$ even if the gaseous fraction exceeds half of the disk mass. However, in the most massive disks we find a threshold value $(0.2)$ of the gas fraction able to destroy the bar.

The stellar bar strength is enhanced by the gas and in the more massive disks higher gas fractions increase the bar pattern speed.
\end{abstract}

Key words. galaxies: spiral - galaxies: structure - galaxies: evolution - galaxies: halos - galaxies: kinematics and dynamics

\section{Introduction}

In a pioneering paper Berentzen et al. (1998) presented the numerical results describing the impact of a gas fraction inside a stellar disk on the stellar orbits and on the whole bar instability. Their model galaxy containing stars and gas was compared with a pure stellar model, subject to the same initial conditions, chosen to produce the development of a strong stellar bar. They showed that dynamical instabilities become milder in the presence of the gas component, and therefore a weakening of the bar itself is observed as a gradual process. Moreover, the overall evolution is accelerated because of the higher central mass concentration and the resulting decrease in the characteristic dynamical time.

The gas behaviour in disk galaxies and its connections with the bar feature has been studied in several papers (Friedli \& Benz 1993; Fux 1999; Berentzen et al. 2001; Athanassoula 2002; Bottema 2003; Bournaud et al. 2005; Michel-Dansac \& Wozniak 2006; Stinson et al. 2006). However in all these works the evolution of the disk or of the disk-halo simulated system arises in an isolated framework, outside the cosmological scenario, even if progressive efforts to improve the model have been performed. These efforts account for the more recent knowledge coming from the cosmological hierarchical clustering scenario of structure formation about density distribution and concentration of the dark matter (DM) halos, and have been performed in last years (Curir \& Mazzei 1999; Mazzei \& Curir 2001; Athanassoula \& Misiriotis 2002). For the first time, we analyse the growth and the evolution of the bar instability in stellargaseous disks embedded in a DM halo evolving in a fully consistent cosmological scenario. Our model cannot be viewed as a general galaxy evolution model, since the gradual formation and growth of the stellar disk has not been taken into account.
Thus our work cannot be compared with the more recent papers by Abadi et al. (2003), Governato et al. (2004), Robertson et al. (2004), Sommer-Larsen (2006), where the formation of a realistic disk galaxy within the hierarchical scenario of structure formation in $\Lambda$-dominated cosmologies has been followed selfconsistently. However, our approach allows us to vary parameters like the disk-to-halo mass ratio and the gas fraction inside the disk and to analyse the growth of the bar instability and its dependence on these parameters in a self-consistent cosmological framework for the first time.

Following the pioneering study by Berentzen et al. (1998), we compare the results of these new set of simulations with those of a previous set (Curir et al. 2006) including a pure stellar disk in the same scenario and with the same initial conditions. The plan of the paper is the following: in Sect. 2, we summarise our recipe for the initial disk + halo system fully described in Paper I. In Sect. 3 we present the cosmological simulations and in Sect. 4 we point out our results. Section 5 is devoted to our discussion and conclusions.

\section{Method}

We embed a gaseous and stellar disk inside a cosmological halo selected in a suitable slice of the Universe and follow its evolution inside a cosmological framework: a $\Lambda$ CDM model with $\Omega_{\mathrm{m}}=0.3, \Omega_{\Lambda}=0.7, \sigma_{8}=0.9$ and $h=0.7$, where $\Omega_{\mathrm{m}}$ is the total matter of the Universe, $\Omega_{\Lambda}$ the cosmological constant, $\sigma_{8}$ the normalisation of the power spectrum, and $h$ the value of the Hubble constant in units of $100 \mathrm{~h}^{-1} \mathrm{~km} \mathrm{~s}^{-1} \mathrm{Mpc}^{-1}$.

A detailed description of our method of producing the cosmological scenario where the disk is evolved has been given in Curir et al. (2006). Here we present a short overview of our recipe. Moreover, Curir et al. (2006) showed that the numerical 
resolution does not impair their main result: in pure stellar disks, long living bars are a "natural" outcome of the cosmological scenario.

\subsection{The DM halo}

To select the DM halo, we performed a low-resolution simulation of a "concordance" $\Lambda$ CDM cosmological model. The initial redshift is 20 . From this simulation we identified the DM halos at $z=0$ in the mass ${ }^{1}$ range $0.5-5 \times 10^{1} h^{-1} M_{\odot}$, with a standard friends-of-friends algorithm.

We selected one suitable DM halo with a mass $M \sim$ $10^{11} h^{-1} M_{\odot}($ at $z=0)$. We resample it with the multi-mass technique described in Klypin et al. (2001). The particles of the DM halo, and those belonging to a sphere with a radius $4 h^{-1} \mathrm{Mpc}$, were followed to their Lagrangian position and re-sampled to an equivalent resolution of $1024^{3}$ particles. The total number of $\mathrm{DM}$ particles in the high resolution region is 1216512 , which corresponds to a DM mass resolution of $1.21 \times 10^{6} h^{-1} M_{\odot}$. The high-resolution DM halo was followed to the redshift $z=0$.

After selecting the halo and resampling at the higher resolution the corresponding Lagrangian region, we run the DM simulation, to extract the halo properties in absence of any embedded stellar disk. The mass of our halo at $z=0,1.03 \times 10^{11} h^{-1} M_{\odot}$ corresponds to a radius, $R_{\mathrm{vir}}=94.7 h^{-1} \mathrm{kpc}$, which entails 84720 halo particles. The nearest DM halo ${ }^{2}$ that is more massive than $10^{10} h^{-1} M_{\odot}$ is $\sim 1900 h^{-1} \mathrm{kpc}$ away from the centre of our halo; the less massive one, with a mass of $4.6 \times 10^{7} h^{-1} M_{\odot}$, is $\sim 215 h^{-1}$ kpc away. Moreover, the behaviour of the density contrast, $\delta$, is monotonically decreasing with the radius, and $\delta$ falls below the unity value at $\sim 550 h^{-1}, 450 h^{-1}, 350 h^{-1}$ physical kpc away from the centre of our halo at $z=0, z=1$, and $z=2$, respectively. Therefore, we conclude that the selected halo is living in an underdense environment. From its accretion history (see Curir et al. 2006, Fig. 1) we conclude that our halo undergoes no significant merger whether during the time it hosts our disk, or immediately before. The halo density profile is well-fitted by a Navarro, Frenk, and White (NFW) form (Navarro et al. 1996, $1997)$ at $z \leq 2$. The concentration, $C_{\text {vir }}$, equal to ${ }^{3} R_{\text {vir }} / R_{\mathrm{s}}$, takes a high value, 18.1, confirming that this halo does "form" at quite a high redshift (e.g. Wechsler et al. 2002, for a discussion about the link between concentration and assembly history of the halo). The dimensionless spin parameter of the halo is 0.04 at $z=2$, near to the average one for our cosmological model (Maller et al. 2002).

\subsection{The baryonic disk}

The spatial distribution of particles follows the exponential surface density law: $\rho=\rho_{0} \exp -\left(r / r_{0}\right)$ where $r_{0}$ is the disk scale length, $r_{0}=4 h^{-1} \mathrm{kpc}$, and $\rho_{0}$ the surface central density. The disk is truncated at five scale lengths with a radius $R_{\text {disk }}=$ $20 h^{-1} \mathrm{kpc}$. To obtain each disk particle's position according to

${ }^{1}$ In the following, we refer to the mass as the virial mass i.e. what is enclosed in a sphere with overdensity $\delta=\rho / \rho_{\text {crit }}=178 \cdot \Omega_{\mathrm{m}}^{0.44}$ (Navarro $\&$ Steinmetz 2000).

${ }^{2}$ Halos have been identified using the friends of friends algorithm with a linking length $l=0.15$, i.e. the mean interparticle distances, and with more than 8 particles

${ }^{3}$ We note, however, that $C_{\mathrm{NFW}}$ is defined against $R_{200}$, the radius enclosing a sphere with overdensity equal to 200 times the critical density of the Universe, and not against $R_{\mathrm{vir}}$ as here; therefore, in our cosmological model, it is always $C_{\mathrm{NFW}}<C_{\mathrm{vir}}$. At $z=0$ our halo has $C_{\mathrm{NFW}} \sim 14$.
Table 1. Simulations: initial values.

\begin{tabular}{cccccccc}
\hline \hline$N$ & $M_{\text {disk }}$ & Gas & $M_{\text {DM }}$ & $R_{\text {DM }}$ & $\alpha r_{m}$ & $\frac{v_{\mathrm{m}}}{\left(\alpha G M_{\text {diks }}\right)^{1 / 2}}$ & Halo \\
\hline c1 & 0.33 & 0.1 & 0.64 & 1.94 & 1. & 1.08 & cosm. \\
c2 & 0.33 & 0.2 & 0.64 & 1.94 & 1. & 1.08 & cosm. \\
c3 & 0.33 & 0.4 & 0.64 & 1.94 & 1. & 1.08 & cosm. \\
c4 & 0.1 & 0.1 & 0.64 & 6.4 & 0.9 & 1.68 & cosm. \\
c5 & 0.1 & 0.2 & 0.64 & 6.4 & 0.9 & 1.68 & cosm. \\
c6 & 0.1 & 0.4 & 0.64 & 6.4 & 0.9 & 1.68 & cosm. \\
c7 & 0.1 & 0.5 & 0.64 & 6.4 & 0.9 & 1.68 & cosm. \\
c8 & 0.1 & 0.6 & 0.64 & 6.4 & 0.9 & 1.68 & cosm. \\
i1 & 0.33 & 0.2 & 0.95 & 2.87 & 0.85 & 1.5 & NFW \\
\hline
\end{tabular}

Table 2. Simulations: final results.

\begin{tabular}{cccccccc}
\hline \hline$N$ & $M_{\mathrm{DM}}$ & $R_{\mathrm{DM}}$ & $\epsilon$ & $Q_{\mathrm{b}}$ & $a_{\max }$ & Bulge & Bars in bars \\
\hline $\mathrm{c} 1$ & 0.77 & 2.39 & 0.68 & 0.43 & 8.4 & $\mathrm{y}$ & $\mathrm{n}$ \\
$\mathrm{c} 2$ & 0.78 & 2.4 & 0.1 & 0.01 & $\mathrm{n}$ & $\mathrm{n}$ & $\mathrm{n}$ \\
$\mathrm{c} 3$ & 0.78 & 2.4 & 0.07 & 0 & $\mathrm{n}$ & $\mathrm{n}$ & $\mathrm{n}$ \\
$\mathrm{c} 4$ & 0.73 & 7.43 & 0.58 & 0.32 & 5.8 & $\mathrm{n}$ & $\mathrm{y}$ \\
$\mathrm{c} 5$ & 0.73 & 7.44 & 0.6 & 0.3 & 5.4 & $\mathrm{n}$ & $\mathrm{n}$ \\
$\mathrm{c} 6$ & 0.73 & 7.56 & 0.46 & 0.23 & 5.6 & $\mathrm{n}$ & $\mathrm{n}$ \\
$\mathrm{c} 7$ & 0.73 & 7.6 & 0.46 & 0.2 & 5.4 & $\mathrm{n}$ & $\mathrm{n}$ \\
$\mathrm{c} 8$ & 0.73 & 7.6 & 0.42 & 0.21 & 5.8 & $\mathrm{n}$ & $\mathrm{n}$ \\
$\mathrm{i} 1$ & 1. & 3.03 & $\mathrm{n}$ & $\mathrm{n}$ & $\mathrm{n}$ & $\mathrm{n}$ & $\mathrm{n}$ \\
\hline & & & & & & &
\end{tabular}

the assumed density distribution, we used the rejection method (Press et al. 1986). We used 56000 star particles and 56000 gaseous particles to describe our disk. The (Plummer equivalent) softening length, the same for DM, gas, and star particles, is $0.5 h^{-1} \mathrm{kpc}$ in comoving coordinates. The stellar radial velocity dispersion, $\sigma_{\mathrm{R}}$, is assigned through a Toomre parameter $Q$, initially constant at all disk radii, which is defined as $Q=\frac{\sigma_{\mathrm{R}} K}{3.36 \mathrm{G} \Sigma}$, where $\kappa$ is the epicyclic frequency and $\Sigma$ the surface density of the disk. We plug in a value of $Q: 1.5$, which corresponds to a warm disk. The gas particles share the same velocity field as the stars. The constraint $Q_{\mathrm{g}}>1$, where $Q_{\mathrm{g}}=\frac{v_{\text {sound }} K}{\pi G \Sigma_{\mathrm{g}}}$, as needed for the gaseous disk stability (Friedli \& Benz 1993) is always verified.

We embed the disk in the high resolution cosmological simulation, at redshift 2 , in a plane perpendicular to the angular momentum vector of the halo and in gravitational equilibrium with the potential. Its centre of mass corresponds to the the minimum potential well of the DM halo. The initial redshift corresponds to $10.24 \mathrm{Gyr}$ down to $z=0$ in our chosen cosmology.

\section{Simulations}

We performed eight cosmological simulations of a disk+halo system. We also performed one simulation in an isolated framework, using an NFW halo, to disentangle the effects of the dynamical state of the halo, and in particular of the evolutionary framework, on the growth of the bar instability (Sect. 4.1). We exploited the public parallel $N$-body treecode GADGET-2 (Springel 2005).

The simulations run on the SP4 and CLX computers located at the CINECA computing centre (BO, Italy; grant inato003) and on the OATo Beowulf-class cluster of 32 Linux-based PC (16 processor AMD K7, clock $700 \mathrm{MHz}$, and 16 Pentium 4, clock $1700 \mathrm{MHz}$ ) at the Osservatorio Astronomico di Torino.The main parameters and the initial properties of our set of simulations are listed in Table 1.

A global stability criteria for the stellar bar instability in a disk galaxy is the one analysed in Efstathiou et al. (1982). In this paper the parameters $\alpha r_{\mathrm{m}}$ and $\frac{v_{\mathrm{m}}}{(\alpha M G)^{1 / 2}}$ (where $v_{\mathrm{m}}$ is the 

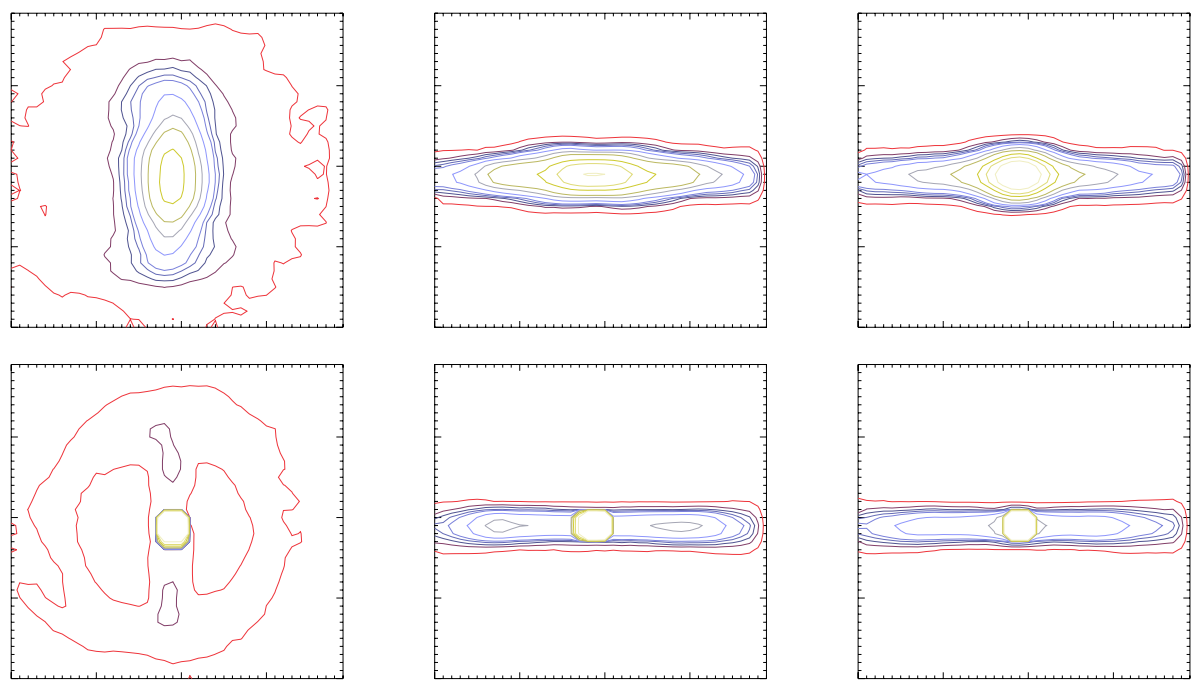

Fig. 1. Face-on, edge-on, and side-on isodensity contours (from left to right) of simulation $\mathrm{c} 1$ at $z=0$ (see text); the top panel shows the stellar component, the bottom panel the gaseous component. Spatial resolution is always $0.5 h^{-1}$ physical $\mathrm{kpc}$ and the box size is 40 times the spatial resolution. Contours are computed at 11 fixed levels ranging from $2 \times 10^{-4}$ to 0.015 in terms of density fraction of stars (gas) within the spatial resolution to the total star (gas) density in the map. maximum value of the disk rotational curve, $r_{\mathrm{m}}$ the corresponding radius, $\alpha=r_{0}{ }^{-1}$, and $M$ is the disk mass) are defined. Efstathiou et al. (1982) state the criterion $\frac{v_{\mathrm{m}}}{(\alpha M G)^{1 / 2}} \geq 1.1$ over the range $0.1 \leq \alpha r_{\mathrm{m}} \leq 1.3$ for a disk to be stable to bar formation. The values of these parameters are also reported in Table 1. In this table we present: in the first column the simulation number and simulation type (c: cosmological simulation, $\mathrm{i}$ : isolated simulation), the mass of the disk in code units (i.e. $5.9 \times 10^{10} M_{\odot}$ ) (Col. II), the fraction of gas, i.e. gas-to-disk mass ratio (Col. III), the DM mass inside the disk radius in code units (Col. IV), the halo-to-disk mass ratio inside the disk radius (Col. V), the Efstathiou et al. (1982) parameters (Cols. VI and VII), the type of halo used (Col. VIII). Simulation i1 corresponds to the evolution of the same disk as in simulation c2, but we embedded it in an NFW halo with the same virial mass and particle number as in our cosmological halo at $z=0$ (see Curir et al. 2006 for more details). Such a halo is spherical, isotropic, and is in gravitational equilibrium. The procedure used to construct this halo is described by Hernquist (1993).

All the results in Table 2 can be compared with those of the homologous simulations performed with a pure stellar disk, as presented in Curir et al. (2006). We repeated, indeed, one of their simulations with the GADGET-2 code, since the time-stepping criterion we used is no longer present in the current version of the code. A comparison with the previous run does not show any significative change. In such a paper we also performed several numerical tests on the stability of the results. In Table 2 we present, as final values, the DM inside the disk radius (code units) (Col. II), the halo-to-disk mass ratio inside the disk radius (Col. III), the maximum ellipticity at $z=0$ (Col. IV): strong bar (Mazzei \& Curir 2001) require $\epsilon \geq 0.4$, the bar strength according to Combes \& Sanders (1981) (Col. V) (stronger bars correspond to higher $Q_{\mathrm{b}}$ values), the major axis (physical kpc) corresponding to the maximum bar strength (Col. VI), the morphology of the inner region of the disk (Col. VII), peculiar features inside the disk (Col. VIII)

Here we summarise our findings:

- A simulation performed with a mass resolution increased by a factor 8 , i.e. with a force resolution increased by a factor 2 (for a disk-to-halo mass ratio 0.1; see Sect. A.2 in Curir et al. 2006), gives rise to a long-living bar (10 Gyr old) as in the case of the lower mass and force resolution. Note that in this test the mass and force resolution are respectively $M_{\mathrm{DM}}^{\mathrm{part}} \approx$ $2 \times 10^{5} h^{-1} M_{\odot}$ and $\epsilon \approx 250 h^{-1}$ pc, thus comparable to the resolution of recent works on galaxy formation simulations, (Sommer-Larsen 2006; Stinson et al. 2006; Governato et al. 2007).

- Embedding adiabatically a classically stable stellar disk in an isolated DM halo having a NFW profile, while keeping the mass and force resolution constant, brings to no bar instability, thus ruling out the possibility that the embedding procedure is the main driver of the bar instability (see Sect. 5 in Curir et al. 2006).

- Varying the stellar softening, at fixed mass resolution, from $0.36 h^{-1} \mathrm{kpc}$ to $0.65 h^{-1} \mathrm{kpc}$ does not result in any significant changes as far as the bar instability is concerned (see Sect. A.3 in Curir et al. 2006).

- The same holds when the stellar mass resolution is reduced by a factor of $1 / 6$ and increased up to 10 times the value adopted in our set of simulations (see A.2. in Curir et al. 2006).

These results guarantee that, in the dynamical range currently reached with tree-code $N$-body simulations, no mayor numerical shortcoming is expected, at least from the point of view of the gravitational evolution. Moreover, as a final test, we re-run simulation c2 using two times more gaseous particles, 112000 instead of 56000 . We find that the final bar dissolution and the gas inflow are the same as in the previous case.

\section{Results}

In this section we present the final (i.e. at $z=0$ ) isodensity contours for stars and gas of simulations in Table 1. All these figures have been built up with the same box-size, number of levels, and density contrast (see caption of Fig. 1). We define the value of the ellipticity as a measure of the bar strength, $\epsilon=1-b / a$ (Table 2); a strong bar corresponds to $\epsilon \geq 0.4$.

A more dynamical measure of the bar strength at radius $R$ has been defined by Combes $\&$ Sanders (1981) by using the parameter: $Q_{\mathrm{t}}(R)=\frac{F_{\mathrm{T}}^{\max }(R)}{\left\langle F_{R}(R)\right\rangle}$ where $F_{\mathrm{T}}^{\max }(R)=[\partial \Phi(R, \theta) / \partial \theta]_{\max }$ is the maximum amplitude of tangential force at a given radius $R$, and $\left\langle F_{R}(R)\right\rangle=R\left(\partial \Phi_{0} / \partial R\right)$ is the mean axisymmetric radial force derived from the $m=0$ component of the gravitational potential at the same radius. The maximum value of $Q_{\mathrm{t}}(R)$ provides a measure of the bar strength $Q_{\mathrm{b}}$ for the whole galaxy. We evaluated the components of the gravitational force directly from the masses and the radii of the system produced by the 

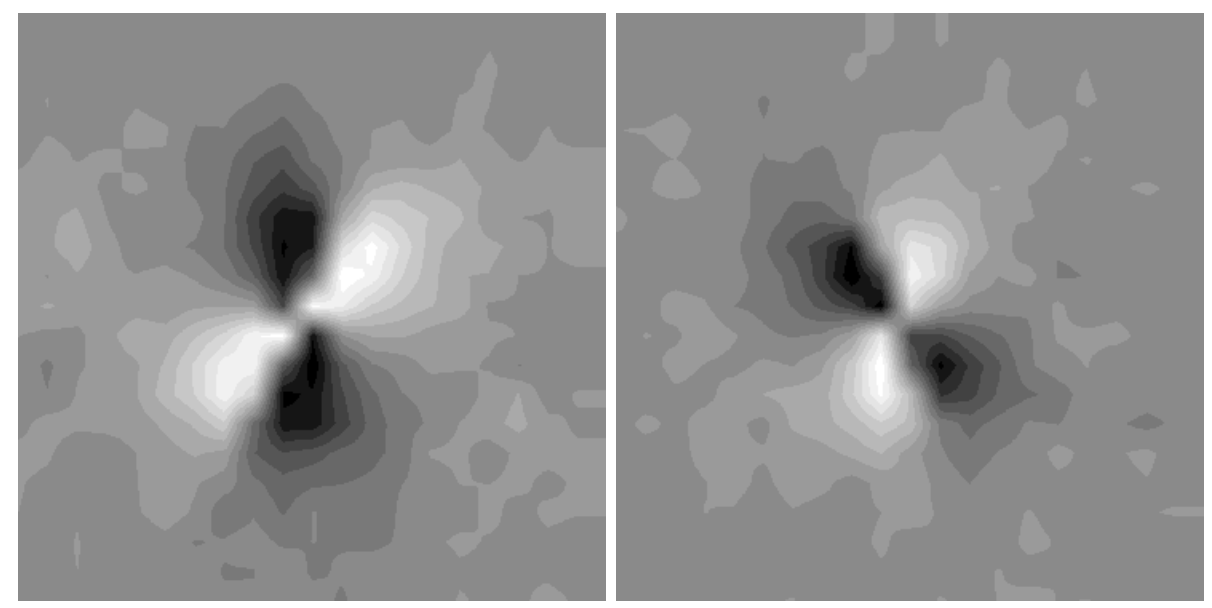

Fig. 2. Maps of the ratios of the axisymmetric radial and tangential forces in the disk plane at $z=0$ for simulation $\mathrm{c} 1$ (left panel) and the analogous case without gas (right panel). Box size and spatial resolution are the same as in Fig. 1.
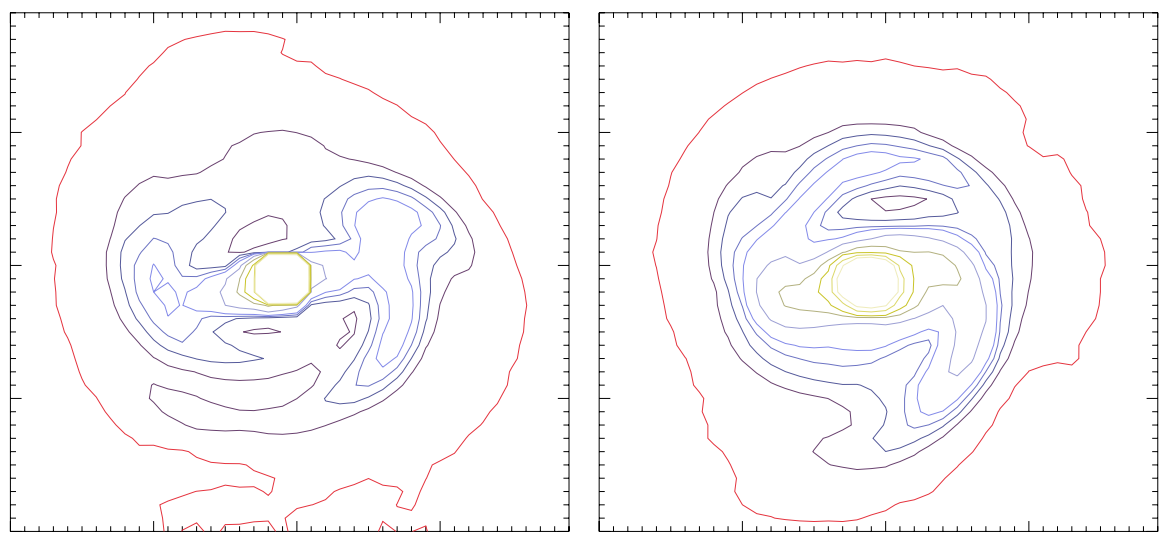

Fig. 3. Gas isodensity contours, $x-y$ projection, of simulation c1 (left panel) and c2 (right panel) at $z=1$. simulations. We then represented the matrix $Q_{\mathrm{t}}(i, j)=\frac{F_{\mathrm{T}}(i, j)}{\left\langle F_{R}(i, j)\right\rangle}$ on a suitable two-dimensional grid in analogy with the method outlined by Buta $\&$ Block (2001). Naming $Q_{\mathrm{b} k}$ the maximum value of $Q_{\mathrm{t}}(i, j)$ in quadrant $k$ of the map, according with Buta \& Block (2001), we define: $Q_{\mathrm{b}}=\frac{\sum_{k=1}^{4} Q_{\mathrm{b} k}}{4}$.

We listed the final values of the parameter $Q_{\mathrm{b}}$ in Table 2 . The values of the bar strength derived with both methods are consistent. In all our cosmological simulations, with the exception of simulations c2 and c3, a stellar bar is still living at $z=0$.

\subsection{Star and gas morphologies}

The strongest and the longest bar arises from simulation c1 (Fig. 1). The "geometrical" strength of this bar, namely the ellipticity, $1-b / a$, evaluated through the isodensity plots, is higher (0.68) than the one measured in the homologous case $(0.52$, Curir et al. 2006). The strength measured through the $Q_{\mathrm{b}}$ parameter is also higher than the corresponding one in the nondissipative homologous case as shown in Fig. 2, which compares the maps of $Q_{\mathrm{t}}(i, j)$ final values of the stellar bars. The enhancement of the stellar bar strength is due to the gaseous bar that is superimposed, i.e coupled, to the stellar one along all the evolution (see Fig. 1, bottom panel).

In the simulation c2, the gas develops a "grand-design" spiral structure, more evident than in simulation c1 (Fig. 3). This effect can contribute to destroy the stellar bar, in agreement with recent findings by Bournaud et al. (2005). In the same simulation, $\mathrm{c} 2$, the gas produces a barred feature from the earlier stages, lasting until $z \approx 1.2$. After such a redshift, the gaseous central condensation contributes to the bar destruction, according to the claim of Berentzen et al. (1998). These authors show that the growth of a gas mass concentration in the disk dissolves the regular orbits in the stellar bar. In this simulation, the stellar bar decreases its strength and disappears completely at $z \approx 0.15$, whereas in the $\mathrm{c} 3$ case, which corresponds to a larger initial gas fraction, the bar disappears at $z \approx 0.6$. Figure 4 shows the gas and star morphologies of simulation $\mathrm{c} 2$ at $z=0$. Simulation $\mathrm{c} 4$ shows a stronger stellar bar than in Curir et al. (2006) (ellipticity 0.58 from Table 2 instead of 0.3 from their Table 3)and bar-in bar features along the whole evolution until $z=0$ (see Fig. 5). Simulations from $\mathrm{c} 5$ to $\mathrm{c} 8$, which correspond to increasing gas fractions, show the same, but less enhanced, features during the system evolution, so that at $z=0$ no bar-in-bar appear and for simulation c8 (Fig. 6), the final bar is the weaker one. In this set of DM-dominated disk simulations, the action of the increasing gas mass is not enough to destroy the bar. We note that, also in these cases, the gaseous bar is coupled with the bar of the stars from the beginning, however, simulations with the larger gas fraction restore the gaseous disk earlier, so that gas and star distributions decouple from $z=1$ (Fig. 7).

In Fig. 8 we show the behaviour of the ellipticity and of the $Q_{\mathrm{b}}$ as a function of the increasing mass fraction in disks of different masses. The agreement we recover between the trends is remarkable: the dynamical evaluation of the bar strength and the geometrical evaluation of it through ellipticities.

Figure 9 compares the gas and star morphologies of the non-cosmological simulation, i1, with those of the homologous non-dissipative case (simulation i4 in Tables 2 and 3 of 

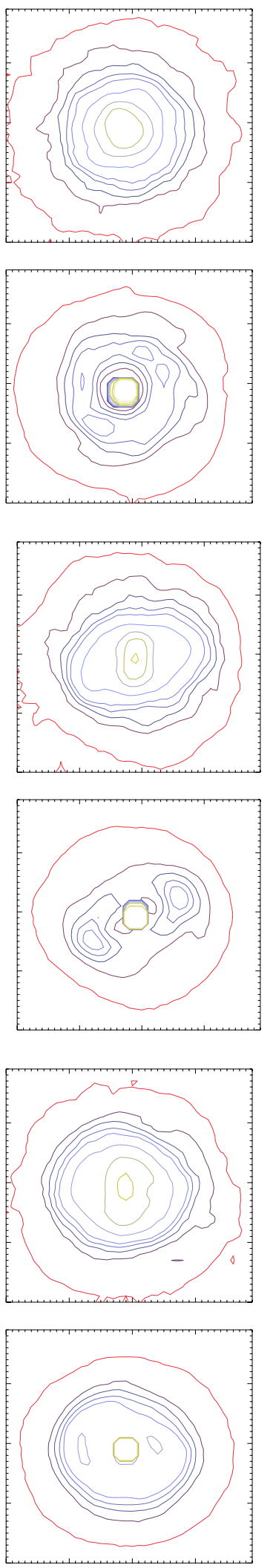
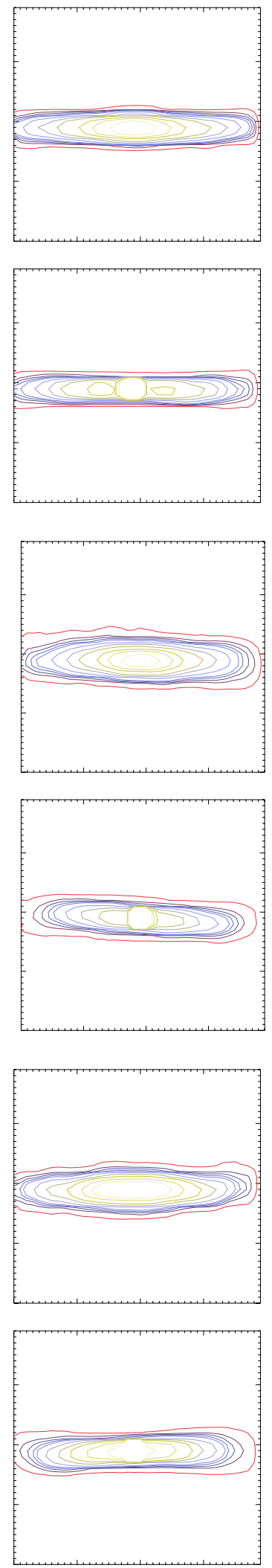
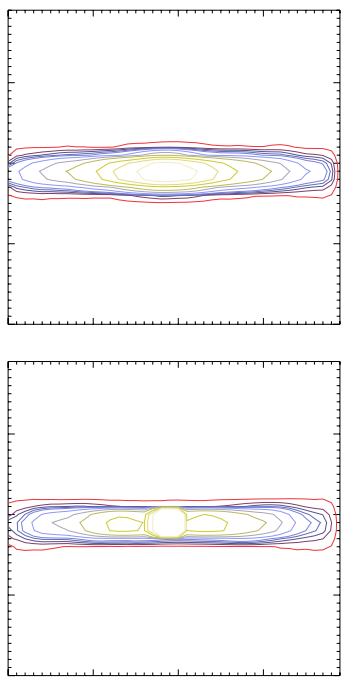

Fig. 4. As in Fig. 1 for simulation c2.
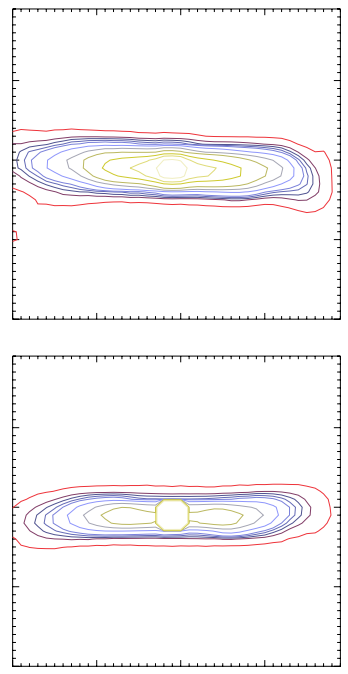

Fig. 5. As in Fig. 1 for simulation c4.
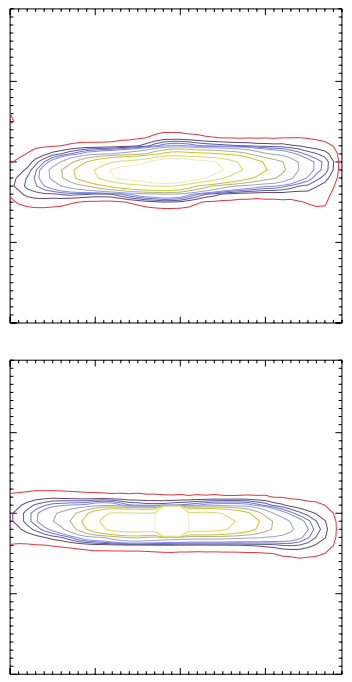

Fig. 6. As in Fig. 1 for simulation c8.

Curir et al. 2006) after 10.24 Gyr. In simulation i1 the bar forms approximately at the same time as in the pure stellar case; however, the presence of the gas destroys the bar after $7.5 \mathrm{Gyr}$ instead of $8.3 \mathrm{Gyr}$ as in simulation c2. Thus the cosmological framework fuels a longer living bar, moreover, when the disk-to-halo mass ratio is 0.33 . In such a framework we find a critical value for the gas fraction, 0.2 , able to destroy the bar.
In the case of DM-dominated disks, all the simulations performed here show a bar feature still present at $z=0$. This is enhanced for the lower gas percentage, 0.1 , and slightly weakened for the higher ones, compared with the corresponding no dissipative case (Curir et al. 2006), but the bar cannot be destroyed by the presence of the gas. 

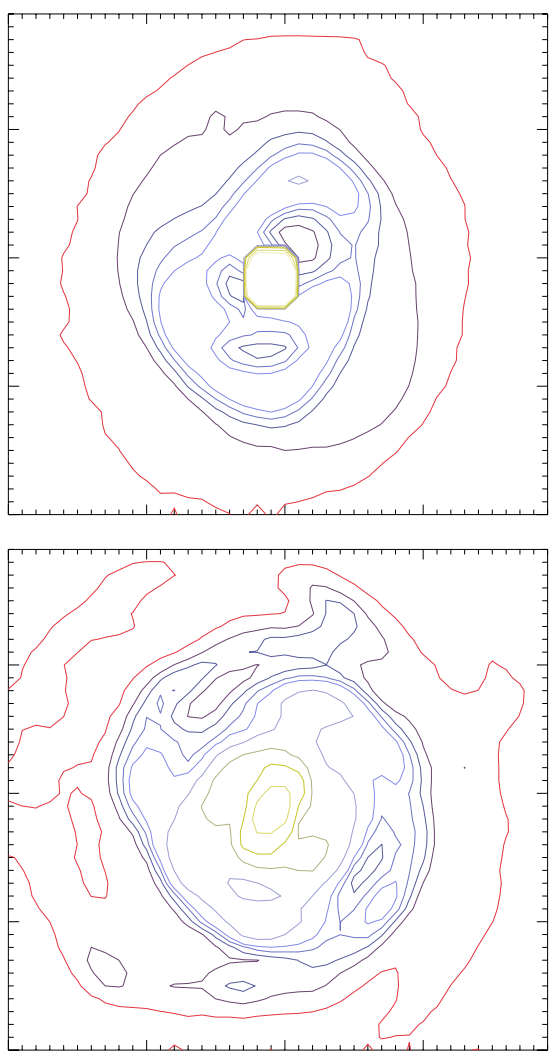
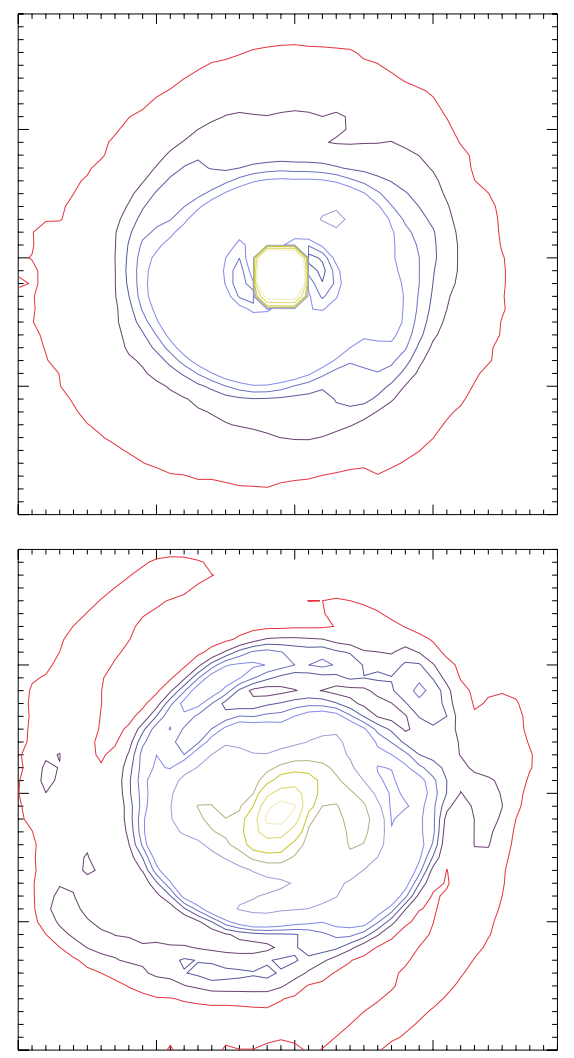

Fig. 7. Gas isodensity contours (top panel), $x-y$ projection, of simulation $\mathrm{c} 4$ (left panel) and $\mathrm{c} 8$ (right panel) at $z=1$; bottom panels show that corresponding contours for stars.

\subsection{Mass inflow and circular velocities}

The gas, initially distributed over all the disk surface with the same density distribution as the stars, evolves by increasing its concentration, depending on the disk-to halo mass ratio and on its initial mass fraction. Both these parameters affect the life and the strength of the bar, as discussed above.

One crucial parameter for monitoring the ability of the central gas condensation to destroy the bar which is indeed the ratio of the central gas mass over the total disk mass, we show in Figs. 10 and 11. Figure 10 for DM-dominated disk simulations shows that the rate of inflow at each radius decreases for higher gas fractions: from $65 \%$ to $37 \%$ at $2 \mathrm{kpc}$ and from $56 \%$ to $35 \%$ at $5 \mathrm{kpc}$ as we go from simulation c4 to $\mathrm{c} 8$. Figure 11, for our more massive disks, shows the same trend but with a stronger decrease: from $75 \%$ to $35 \%$ at $2 \mathrm{kpc}$ and from $66 \%$ to $41 \%$ at $5 \mathrm{kpc}$ as we go from simulation $\mathrm{c} 1$ to $\mathrm{c} 2$. In particular, for simulation $\mathrm{c} 2$, where the gas fraction is able to destroy the bar, the accretion rate at the outer radii becomes quasi stationary after $z=1$ (Fig. 11, middle panel). Since with the weakening of the bar, the gas inflow is no longer forced. In simulation $\mathrm{c} 3$, such behaviour appears earlier (Fig. 11, middle panel), and the bar disappears earlier than in the $\mathrm{c} 2$ case.

The circular velocity, $v_{\text {circ }}$, computed by assuming a spherical mass distribution and therefore having the expression $(G M / r)^{1 / 2}$ where $M$ is the mass of a component of the system, gives a measure of the absolute concentration of this component. In Figs. 12 and 13 (left panels), we show its behaviour at $z=0$ for gas, stars, and DM in simulations c4 and c6. In the same figures (right panels), we also show the behaviour of the $v_{\text {circ }}=r(\partial \Phi / \partial r)$ evaluated from the force field of the stellar and DM components. The difference of these values from the previous ones is due to the deviation from the spherical isotropy of the mass distribution. In particular, the wide spread we observe for the DM component indicates a wide anisotropy, wider at large radii.

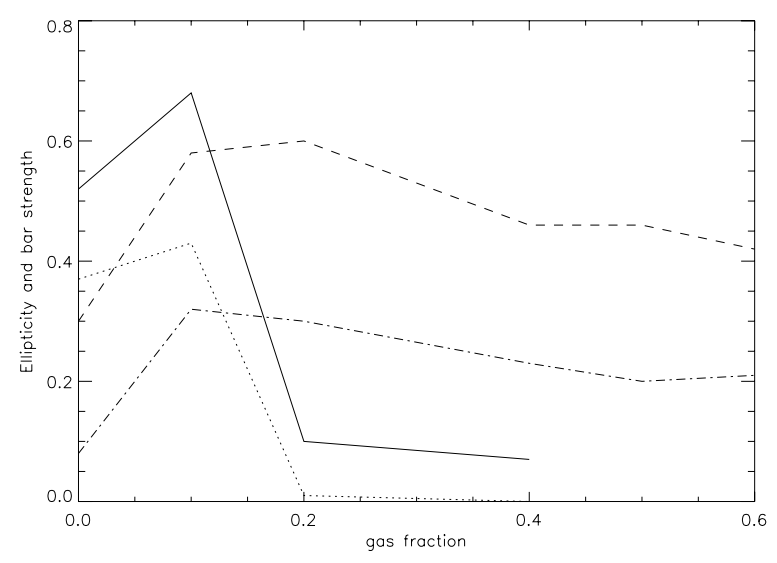

Fig. 8. Behaviour of the bar strength and ellipticity at $z=0$ for our set of cosmological simulations with increasing gas fraction. $Q_{\mathrm{b}}$ (dotted line) and ellipticity (full line) of our more massive disks (i.e. disk-to-halo mass ratio 0.33 ); $Q_{\mathrm{b}}$ (dot-dashed line) and ellipticity (dashed line) of our less massive, DM-dominated, disks (i.e. disk-to-halo mass ratio 0.1).

The impact of a central mass concentration (CMC) on the bar evolution has been discussed in detail by Shen \& Sellwood (2004). Their study is not directly comparable to ours, since the growth of the CMC is modelled with an analytic potential instead of being the result of gas inflow like in our simulations. In their work it was shown that the destruction power depends both on the CMC, given as a fraction of the disk mass, and on the "softening" of its potential, $r_{\mathrm{s}}$, a parameter that controls the compactness of the CMC. Shen \& Sellwood (2004) find that for a hard CMC (namely with $r_{\mathrm{s}}$ very small) only a few percent of the disk mass is able to destroy the bar, whereas for a soft $\mathrm{CMC}$ $\left(r_{\mathrm{s}}=0.3 \mathrm{kpc}\right)$ a fraction larger than 0.1 succeeds. In our scenario, $r_{\mathrm{s}}$ qualitatively translates into the radius, $r_{\mathrm{g}}$, where we set the evaluation of the gas mass concentration. We deduce that, 

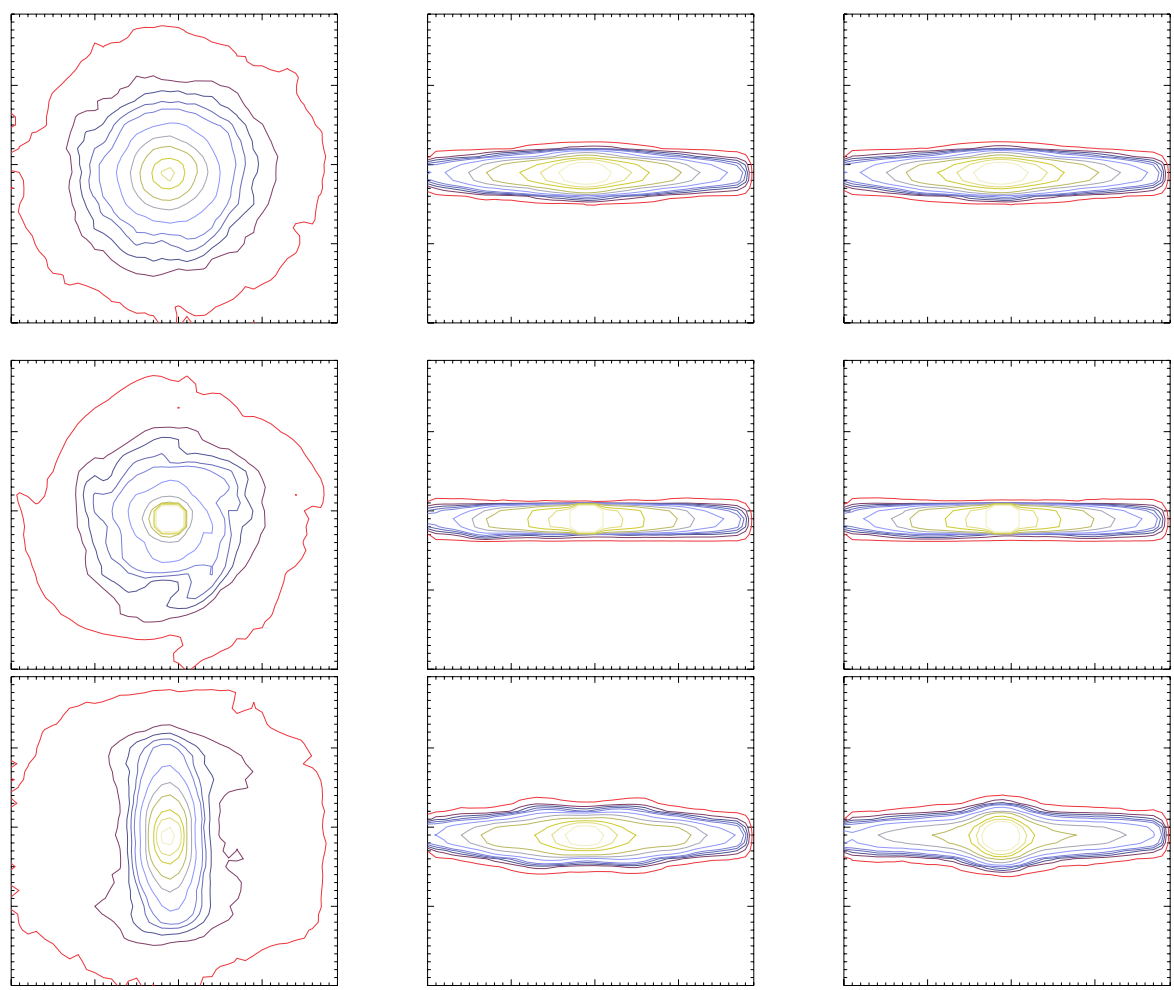

Fig. 9. Isodensity contours of simulation i1 at 10.24 Gyr (top and central panels) compared with the stellar isodensity contours of the same case without gas (bottom panel, simulation $\mathrm{c} 4$ of Curir et al. (2006)) at $z=0$. for our more massive disks (i.e. when $R_{\mathrm{DM}}=1.94$ in Table 1), a gas mass greater than $9 \%$ of the disk mass inside $r_{\mathrm{g}}=2 \mathrm{kpc}$ is needed to destroy the bar. However for DM dominated disks, (i.e. with $R_{\mathrm{DM}}=6.4$ in Table 1 ) a value of $18 \%$ of the disk mass in the same $r_{\mathrm{g}}$ cannot destroy the bar. Therefore, our cosmological simulations indicate that also the value of $R_{\mathrm{DM}}$ is a crucial parameter in this play.

\subsection{Bar pattern speed}

The bar pattern speed, $\Omega_{\mathrm{p}}$ is the angular speed of the the barlike density wave as viewed from an inertial frame. Here it is evaluated following the position angle of the major bar axis during the disk evolution, where the major axis is selected with the same criterion E1 quoted in Michel-Dansac \& Wozniak (2006), namely as the radius where the ellipticity profile reaches a maximum. In Fig. 14 the values of $\Omega_{p}$ as a function of the redshift are shown for the two sets of our simulations: the more massive disks and the lighter disks. Since the behaviour of $\Omega_{p}$ is rather noisy, we also provided the error bars calculated with the running average method; i.e. they are obtained averaging three consecutive values and computing their standard deviations. This error bar is more representative of the fluctuations of the quantity $\Omega_{p}$ than of the real errors on the measurements. On the other hand, the uncertainty in the measure of the position angle of the semimajor axis is always less than $10^{\circ}$. This limit leads to a maximum error of $6 \%$ in the evaluation of $\Omega_{\mathrm{p}}$. The error bar related to such a maximum error is presented at the bottom of each panel in Fig. 14. For the more massive disks, the stronger decrease is observed in simulation $\mathrm{c} 1$. This is also the simulation with the longer and the stronger stellar bar (see Table 2), usually coupled with the higher decrease in the pattern speed during the evolution (Athanassoula 2002). Simulation c2 shows the same trend, the decrease in $\Omega_{\mathrm{p}}$ is slightly lower, and the bar disappears before $z=0$, as in simulation $\mathrm{c} 3$. However in such a simulation, the pattern speed increases by decreasing the redshift. We derive that the increasing gas fraction in the more massive disks produces a speeding up of the stellar bar pattern since the presence of gas both shortens the bar and decreases its ellipticity. Moreover, such behaviour is consistent with the findings of Berentzen et al. (1998), i.e. an accelerated evolution of the whole system when the gas is included.

On the other hand, the DM-dominated disks show the strongest decrease in $\Omega_{p}$ with the redshift. The lower dynamical support of these light disks compared to the more massive cases indeed enhances the role of the gas, which slows down the pattern speed more quickly.

\section{Discussion and conclusions}

We have presented eight cosmological simulations with the same disk-to-halo mass ratios as in Curir et al. (2006). In order to study the impact of the gaseous component, we here included and varied its percentage inside disks with different disk-to-halo mass ratios.

Between the more massive disks, simulation $\mathrm{c} 1$, with the lower gas percentage, shows a long-lasting bar, 10 Gyr old, stronger than the one developed in the pure stellar case. In simulation c2, a gas percentage of $20 \%$ appears as a threshold value for the bar life as far as this disk mass is concerned. In such a simulation, indeed, the gas is able to destroy the bar at the very end of the evolution $(z=0.15)$. By increasing the gas percentage, as in simulation c3, the bar disappears earlier than in c2 (at $z=0.6$ ). We find that a gaseous mass concentration equal to $9 \%$ of the total mass of the disk inside a radius of $2 \mathrm{kpc}$ is a lower limit for the bar dissolution. In our more massive disks, where the baryons gravitational field is comparable to that of the DM halo (i.e. disk-to-halo mass ratio 0.33 ), we find a threshold value for the gas fraction, 0.2, able to destroy the bar.

The gas shows a trend to a wider distribution by increasing its fraction, for a given disk mass. Moreover, for the same gas fraction, the mass inflow is stronger in more massive disks. 

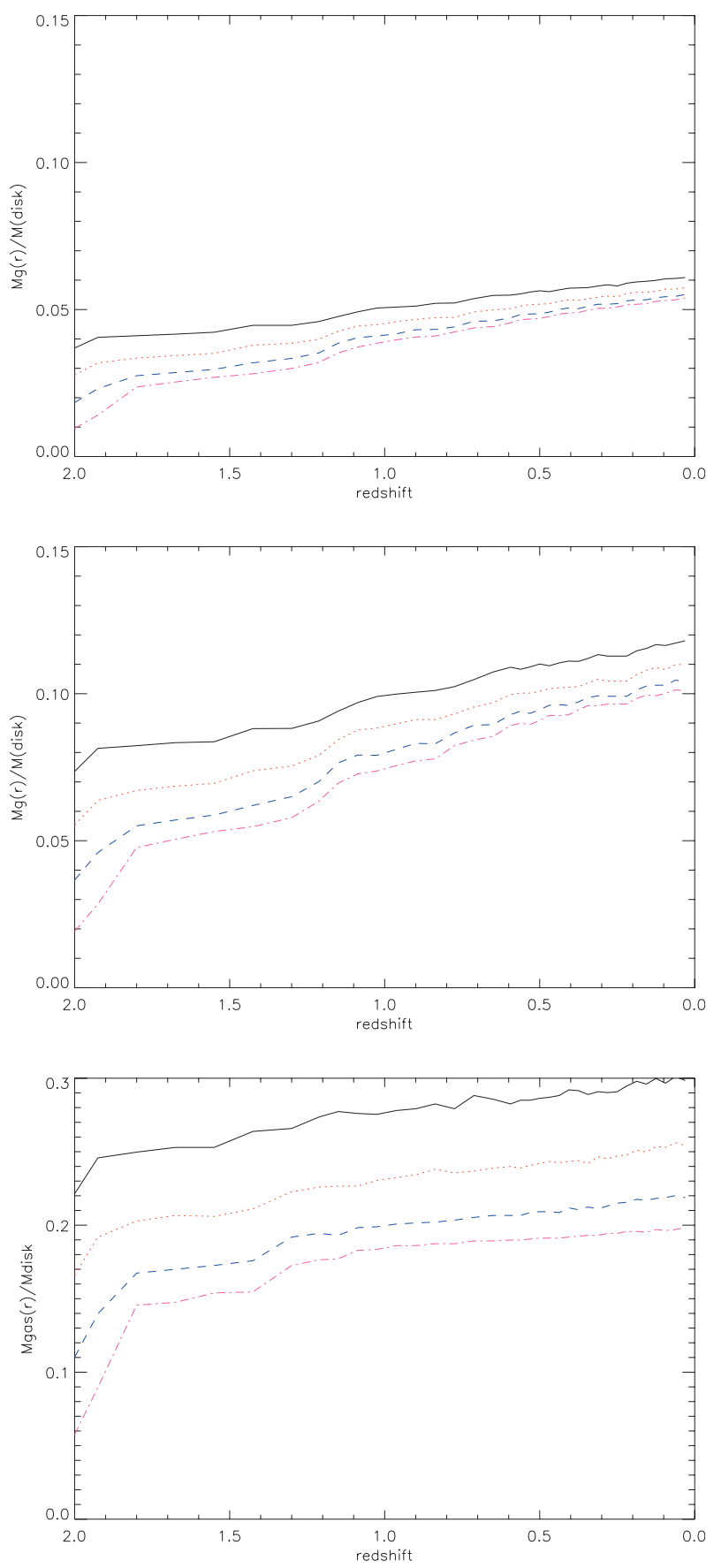

Fig. 10. Gas-to-disk mass ratio at different disk radii: $2 \mathrm{kpc}$ (magenta dashed-dotted line), $3 \mathrm{kpc}$ (blue dashed line), $4 \mathrm{kpc}$ (red dotted line), and $5 \mathrm{kpc}$ (black full line) as functions of redshift for simulation c4 (top panel), c5 (middle panel), and c8 (bottom panel).

These effects contribute to weakening the bar, which in our cosmological framework, however, cannot be destroyed in DMdominated disks. In these cases, indeed, the cosmological evolution of the DM halo drives the growth of the bar instability so that the presence of the gas appears as a second-order effect on its evolution.

The DM-dominated disks indeed show a behaviour that is strongly driven by the cosmology as in Curir et al. (2006). In these cases: i) we do not find any value for the gas fraction, in the range $0.1-0.6$, able to destroy the bar; ii) even a high value of central gas concentration does not succeed in dissolving the bar. The bars in these disks are not a classical product of the
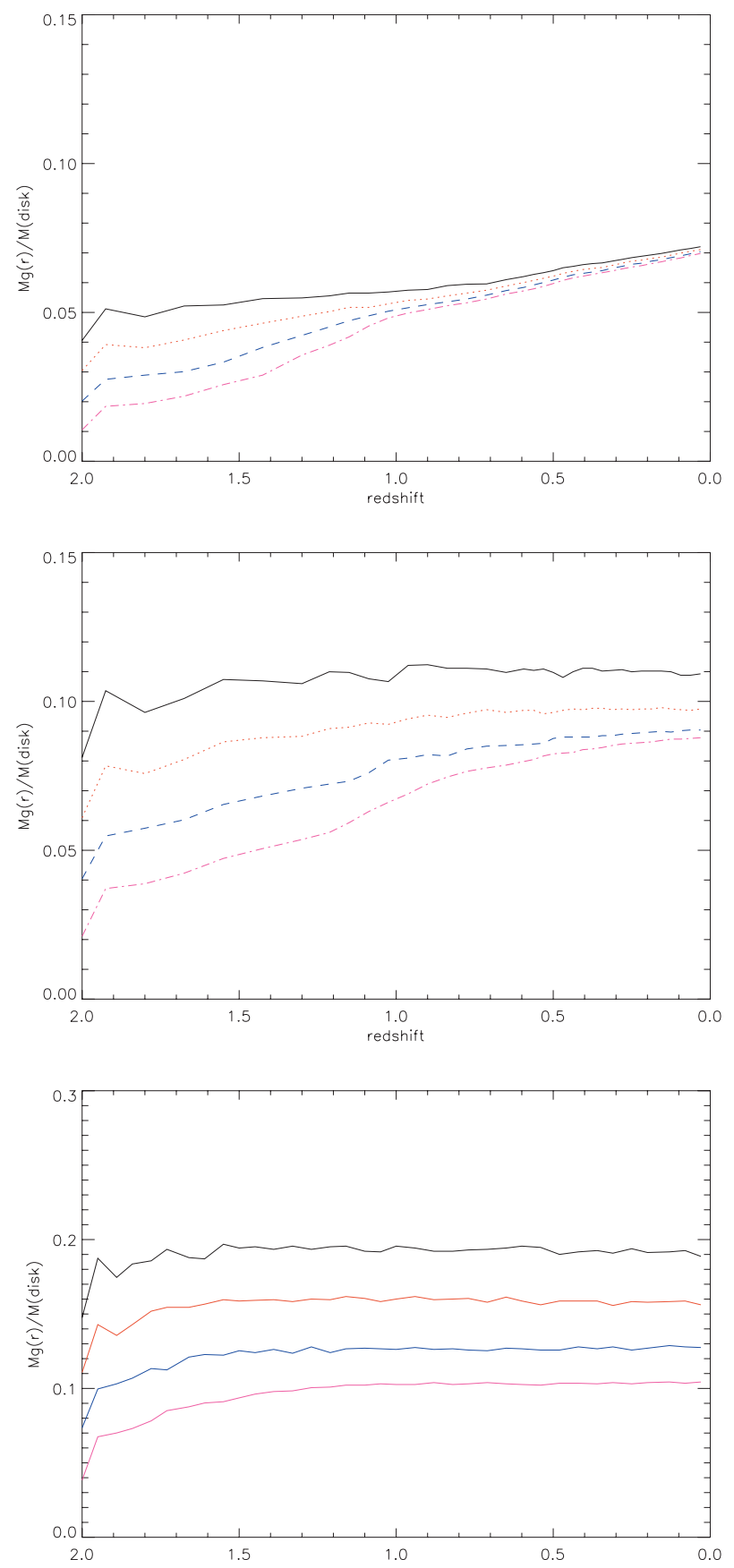

Fig. 11. As in Fig. 10 for simulation $\mathrm{c} 1$ (top panel), c2 (middle panel), and $\mathrm{c} 3$ (bottom panel).

self-gravity, which is very weak, or of angular momentum exchanges, since the disk rotates very slowly; but they are features that strongly depend on the dynamical state and evolution of the cosmological halo, therefore the classical results emphasising the gas impact obtained outside the cosmological scenario are no longer applicable. This confirms the results of Curir et al. (2006), where in the DM-dominated disks the bar feature is triggered and maintained by the cosmological characteristics of the halo, namely its triaxiality and its dynamical state.

Therefore the whole set of cosmological simulations we present here suggests a play between three parameters that drive the bar formation and dissolution in cosmology: the gas fraction inside a suitable disk radius, $r_{\mathrm{g}}$, the $r_{\mathrm{g}}$ value itself, and the haloto-disk mass ratio inside the disk radius. 

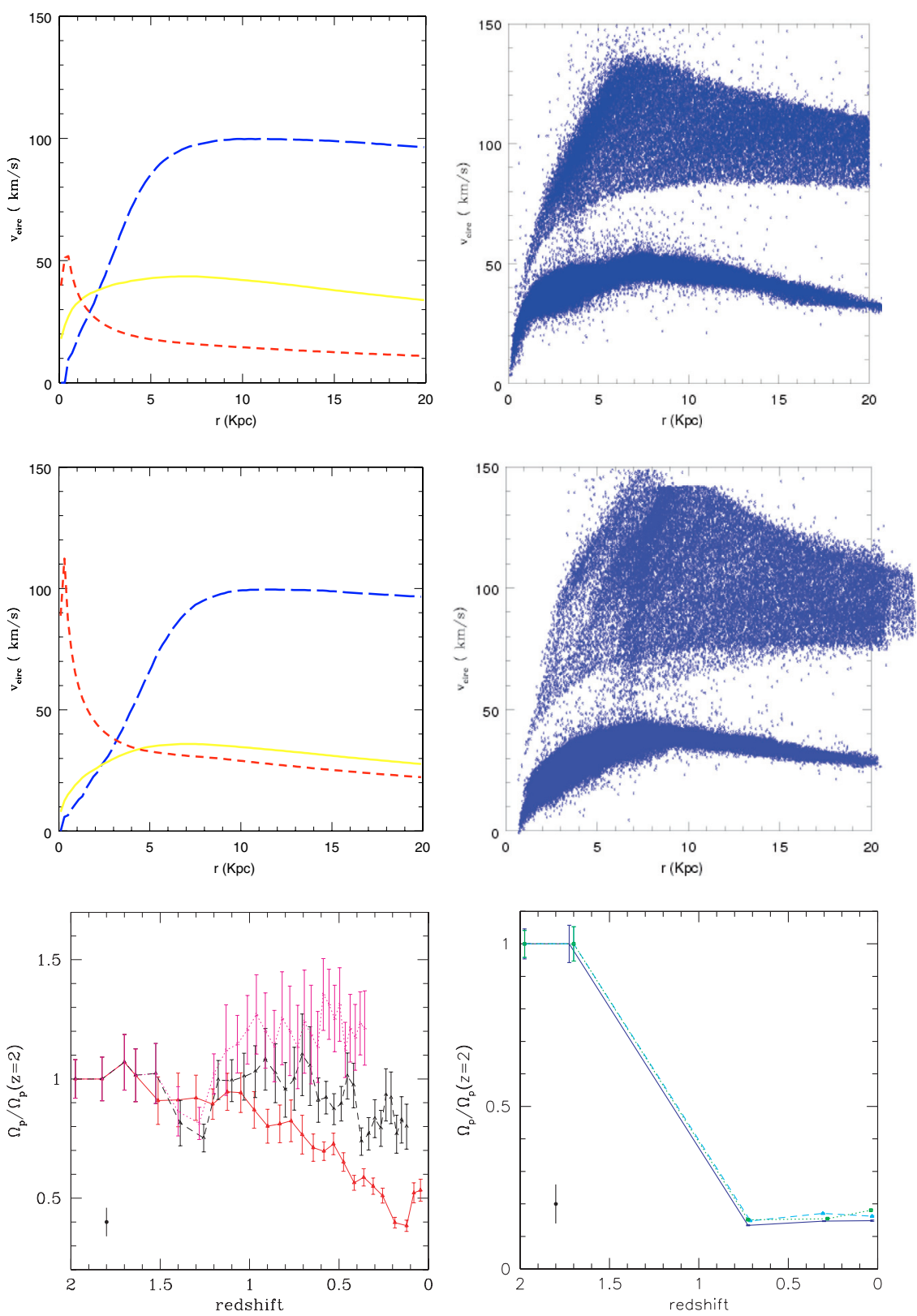

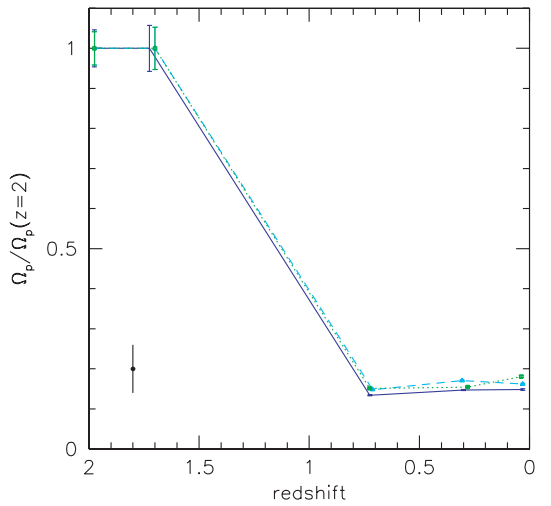

Fig. 12. Left panel: circular velocities obtained assuming a spherical mass distribution of gas (red dashed line), stars (yellow continuous line), and DM (blue long dashed line) at $z=0$ for simulation $\mathrm{c} 4$. Right panel: circular velocities deduced from the radial force (upper curve: dark matter, lower curve: stars) at $z=0$ for simulation $\mathrm{c} 4$.

Fig. 13. The circular velocities as in Fig. 12 for simulation $\mathrm{c} 6$.
Fig. 14. Evolution of the bar pattern speed with the redshift. Left panel: simulation c1 (red full line), c2 (black dashed line), c3 (magenta dotted line). Right panel: simulations c4 (blue full line), c5 (light blue dotted line), and $c 6$ (green dashed line). The error bars on the curves are obtained with the running average method, and the error bar at the bottom of each panel represents the uncertainty in the measure of the position angle of the bar (see text).
Acknowledgements. Simulations were performed on the CINECA IBM SP4 computer, thanks to the INAF-CINECA grants cnato43a/inato003 "Evolution of disk galaxies in cosmological contexts", and on the Linux PC Cluster of the Osservatorio Astronomico di Torino. We wish to acknowledge the useful discussions with: L. Athanassoula, I. Berentzen, F. Bourneaud, E. D’Onghia, A. Klypin \& V. Springel.

\section{References}

Abadi, M. G., Navarro, J. F., Steinmetz, M., \& Eke, V. R. 2003, ApJ, 591, 499 Athanassoula, E. 2002, in Disks of Galaxies: Kinematics, Dynamics and Peturbations, ed. E. Athanassoula, A. Bosma, \& R. Mujica, ASP Conf. Ser. 275,141

Athanassoula, E., \& Misiriotis, A. 2002, MNRAS, 330, 35

Berentzen, I., Heller, C. H., Fricke, K. J., \& Athanassoula, E. 2001, Ap\&SS, 276, 699

Berentzen, I., Heller, C. H., Shlosman, I., \& Fricke, K. J. 1998, MNRAS, 300, 49

Bottema, R. 2003, MNRAS, 344, 358

Bournaud, F., Combes, F., \& Semelin, B. 2005, MNRAS, 364, L18

Buta, R., \& Block, D. L. 2001, ApJ, 550, 243

Combes, F., \& Sanders, R. H. 1981, A\&A, 96, 164

Curir, A., \& Mazzei, P. 1999, A\&A, 352, 103
Curir, A., Mazzei, P., \& Murante, G. 2006, A\&A, 447, 453

Efstathiou, G., Lake, G., \& Negroponte, J. 1982, MNRAS, 199, 1069

Friedli, D., \& Benz, W. 1993, A\&A, 268, 65

Fux, R. 1999, A\&A, 345, 787

Governato, F., Mayer, L., Wadsley, J., et al. 2004, ApJ, 607, 688

Governato, F., Willman, B., Mayer, L., et al. 2007, MNRAS, 374, 1479

Hernquist, L. 1993, ApJS, 86, 389

Klypin, A., Kravtsov, A. V., Bullock, J. S., \& Primack, J. R. 2001, ApJ, 554, 903

Maller, A. H., Dekel, A., \& Somerville, R. 2002, MNRAS, 329, 423

Mazzei, P., \& Curir, A. 2001, A\&A, 372, 803

Michel-Dansac, L., \& Wozniak, H. 2006, A\&A, 452, 97

Navarro, J. F., \& Steinmetz, M. 2000, ApJ, 538, 477

Navarro, J. F., Frenk, C. S., \& White, S. D. M. 1996, ApJ, 462, 563

Navarro, J. F., Frenk, C. S., \& White, S. D. M. 1997, ApJ, 490, 493

Press, W. H., Flannery, B. P., \& Teukolsky, S. A. 1986, Numerical recipes. The art of scientific computing (Cambridge: University Press)

Robertson, B., Yoshida, N., Springel, V., \& Hernquist, L. 2004, ApJ, 606, 32

Shen, J., \& Sellwood, J. A. 2004, ApJ, 604, 614

Sommer-Larsen, J. 2006, ApJ, 644, L1

Springel, V. 2005, MNRAS, 364, 1105

Stinson, G., Seth, A., Katz, N., et al. 2006, MNRAS, 373, 1074

Wechsler, R. H., Bullock, J. S., Primack, J. R., Kravtsov, A. V., \& Dekel, A. 2002, ApJ, 568, 52 